\title{
Optimisation de la chaîne complète du procédé de moulage par injection métallique
}

\author{
Application à une prothèse de hanche
}

\author{
Ghassane Ayad - Thierry Barriere - Jean-Claude Gelin \\ Institut FEMTO-ST \\ Département de Mécanique Appliquée \\ ENSMM Besançon \\ 24 chemin de l'Epitaphe \\ F-25030 Besançon
}

RÉSUMÉ. Cet article est consacré à l'identification paramétrique et à l'optimisation des phases d'injection et de densification par diffusion à l'état solide du procédé de moulage par injection métallique. La phase d'injection est modélisée et simulée grâce à un modèle d'écoulement biphasique, tandis que les mécanismes de densification par diffusion à l'état solide sont représentés par un modèle macroscopique. La qualité des résultats numériques reposant sur les modèles physiques dépend directement de l'identification des paramètres matériaux pour chacune des étapes. On propose dans l'article une combinaison entre l'optimisation paramétrique de la phase d'injection, l'identification paramétrique du modèle de diffusion et enfin l'optimisation de la phase de densification afin de déterminer de manière optimale les dimensions d'empreintes de moule, de façon à obtenir des composants sans défauts, aux dimensions et propriétés mécaniques souhaitées.

ABSTRACT. This paper is related to the parametric identification and optimization of the injection moulding and sintering stage of the Metal Injection Moulding process. The injection stage is described using a biphasic flow formulation, whereas the sintering mechanism by solid state diffusion is accounted through a viscoplastic type material model. The accuracy of the numerical results of the injection moulding or sintering stages is strongly dependent on the identification of physical parameters entering in the models. In this paper one propose to combine the optimization of the injection moulding stage with the parametric identification of the sintering stage in order to determine the size of the mould cavities in order to get components without defects and with the appropriate resulting geometrical sizes and mechanical properties.

MOTS-CLÉS: moulage par injection métallique, optimisation, surfaces réponses, algorithme génétique, identification paramétrique.

KEYWORDS: powder injection moulding, optimization, response surfaces, genetic algorithm, and parametric identification.

DOI:10.3166/REMN.17.397-422 @ 2008 Lavoisier, Paris

REMN - 17/2008. Optimisation des procédés de mise en forme, pages 397 à 422 


\section{Introduction}

On propose dans ce papier le développement d'un optimiseur complet pour le procédé de moulage par injection de poudres, avec comme but la détermination automatique par simulation et optimisation des dimensions d'empreintes de moule, des paramètres de procédés et des caractéristiques physiques des mélanges de façon à obtenir des composants aux dimensions géométriques et propriétés mécaniques souhaitées (Ayad, 2006).

Le moulage par injection métallique (MIM) et plus généralement le moulage par injection de poudres (MIP) consiste en quatre étapes.

Dans un premier temps, les poudres métalliques ou céramiques sont mélangées à un ensemble de polymères thermoplastiques (le liant). Le mélange ainsi produit est souvent nommé «feedstock ». Au cours de la deuxième étape, le mélange est moulé par injection en utilisant les technologies standard de moulage par injection des polymères. Ensuite, le liant est évacué après démoulage lors de la phase de déliantage. Le déliantage peut être réalisé soit par voie thermique, par dissolution aqueuse ou bien encore par dissolution thermochimique. Enfin, le squelette de poudres est rigidifié par densification thermique par diffusion à l'état solide pour réaliser la pièce à la forme souhaitée.

Ce procédé combine donc les technologies du moulage par injection des polymères thermoplastiques, ainsi que les technologies de frittage de poudres en phase solide. Ses caractéristiques sont déterminées par chacune des quatre étapes du procédé (Barriere, 2005).

Le MIP permet d'obtenir des composants de formes très complexes avec des matériaux difficiles, voire impossibles à mettre en forme par d'autres technologies (German, 1997). Ces composants peuvent posséder d'excellentes propriétés mécaniques, fonction des constituants initialement employés.

A priori, tous les matériaux métalliques ou céramiques peuvent être employés. Il convient cependant d'effectuer une étude préalable sur la nature des liants à utiliser. Ceux-ci doivent être capables de véhiculer les poudres lors de l'injection et de ne pas les contaminer chimiquement durant la phase de déliantage.

L'injection permet également des cadences plus élevées que bon nombre d'autres technologies, dont en particulier l'usinage. Cependant, ce procédé n'est actuellement rentable que pour la production en moyennes et grandes séries, compte tenu de l'importance des investissements nécessaires et de la complexité du procédé.

A titre d'exemple, ce procédé est utilisé pour la fabrication de composants en aciers inoxydables, en alliages de titane ou encore en céramiques. Du point de vue des applications, des composants peuvent être produits pour la connectique, le biomédical, la lunetterie, l'horlogerie, la domotique, l'industrie du luxe, l'aéronautique et l'industrie automobile (German, 1997). 
Si le MIP possède les nombreux avantages mentionnés ci-avant, le fait que le MIP comporte plusieurs étapes pouvant influencer la forme et les propriétés finales des composants est l'un des inconvénients majeurs du procédé. En effet, pour obtenir des composants de bonne qualité, chaque étape doit être rigoureusement contrôlée.

La phase de mélangeage et en particulier l'homogénéité du mélange en résultant est très importante pour l'obtention de composants de bonne qualité. Ainsi, un mélange pauvre en liant conduit généralement à une viscosité trop importante du mélange et à l'apparition de bulles. Par contre un excès de liant entraîne une mauvaise répartition des particules métalliques dans les pièces injectées. Cela se traduit par un effondrement de la pièce pendant le déliantage ou par des retraits non hétérogènes durant la densification.

Dans l'hypothèse d'un mélange homogène possédant une bonne répartition de liant, la phase d'injection est déterminante pour fabriquer des pièces satisfaisant les critères souhaités. De cette étape dépend la forme finale de la pièce après densification, ainsi que l'apparition ou non de défauts sur la pièce finale. Parmi les défauts les plus connus et les plus préjudiciables, il y a les phénomènes de ségrégation (Gelin et al., 2004), de jets en parois ou jetting (Piccirillo et al, 1991 ; Dvorak et al., 2005), de mauvaise jonction de fronts, de vides et bulles...

Pour éviter ces défauts, un réglage optimal des paramètres d'injection est nécessaire. Généralement les paramètres contrôlés sont la pression d'injection, la température du moule, la température du mélange. Les poudres (métalliques ou céramiques) et le liant sont naturellement choisis en fonction des propriétés d'usage de la pièce désirée.

La phase de densification est l'étape qui détermine les caractéristiques de comportement matériel de la pièce finale ainsi que sa forme finale. Les défauts pouvant apparaitre sont entre autres le retrait anisotrope, la formation de cloques ou de fissures, les distorsions de la pièce, les répartitions hétérogènes de densité. Les paramètres de contrôle du procédé sont le cycle de montée en température, le temps de densification, les réactions chimiques entre les composants du mélange et l'atmosphère (Song et al., 2005). Mais les défauts survenant au cours de la densification peuvent également être inhérents aux phases précédentes. Ceci complique alors l'identification des causes des problèmes constatés.

\section{Modélisation des étapes d'injection et de densification en moulage par injection de poudre}

\subsection{Modélisation et simulation de l'étape d'injection}

Pour simuler la phase d'injection du mélange poudres et liant, un modèle biphasique a été proposé et utilisé. Ce modèle considère l'écoulement de deux phases distinctes : une phase solide considérée comme un fluide visqueux et un liant 
fluide. L'interaction entre les deux phases et l'incompressibilité du mélange sont prises en compte pour la description de l'écoulement du mélange poudre+liant. Cette théorie est bien adaptée à la simulation des écoulements de fluides visqueux fortement chargés en particules de poudre, elle est par conséquent bien adaptée au procédé MIM. Chacune des phases possède sa propre loi de comportement et les équations de conservation sont écrites pour chacune des phases. L'interaction entre les deux phases est représentée par un coefficient d'interaction.

Le laboratoire a développé un solveur éléments finis basé sur la résolution des équations de conservation de la masse [1], de la quantité de mouvement ou encore équations de Stokes [2], exprimées sous la forme suivante :

$$
\begin{aligned}
& \frac{\partial \phi_{s}}{\partial t}+\nabla \bullet\left(\phi_{s} \mathbf{V}_{\mathbf{s}}\right)=0 \text { et } \frac{\partial \phi_{f}}{\partial t}+\nabla \bullet\left(\phi_{f} \mathbf{V}_{\mathbf{f}}\right)=0 \\
& \rho_{s} \frac{\partial \mathbf{V}_{\mathbf{s}}}{\partial t}=-\nabla\left(\phi_{s} P\right)+\nabla \bullet \boldsymbol{\sigma}_{\mathbf{s}}^{\prime}+\rho_{s} \mathbf{g}+\mathbf{m}_{\mathbf{s}} \\
& \rho_{f} \frac{\partial \mathbf{V}_{f}}{\partial t}=-\nabla\left(\phi_{f} P\right)+\nabla \bullet \boldsymbol{\sigma}_{f}^{\prime}+\rho_{f} \mathbf{g}+\mathbf{m}_{f}
\end{aligned}
$$

où $\phi_{s}$ et $\phi_{f}$ représentent respectivement la fraction volumique de la phase solide et fluide. $\mathbf{V}_{\mathbf{s}}, \mathbf{V}_{\mathbf{f}}$ représentent respectivement les vitesses des phases solide (poudres) et fluide (liant). Les densités de chacune des phases sont définies par les relations $\rho_{s}=\phi_{s} \rho_{s}^{o}$ et $\rho_{f}=\phi_{f} \rho_{f}^{o}$ où $\rho_{s}^{o}$ et $\rho_{f}^{o}$ caractérisent les densités initiales du liant et des poudres supposées constantes, $P$ représente le champ de pression, $\boldsymbol{\sigma}_{\mathbf{s}}^{\prime}$ et $\boldsymbol{\sigma}_{f}^{\prime}$ sont les déviateurs des tenseurs des contraintes partielles de Cauchy des phases solide et fluide et $\boldsymbol{g}$ la gravité, $\mathbf{m}_{\mathbf{s}}=-\mathbf{m}_{\mathbf{f}}$ sont les termes d'interactions qui s'écrivent sous la forme suivante $\mathbf{m}_{\mathbf{s}}=k\left(\mathbf{V}_{\mathbf{f}}-\mathbf{V}_{\mathbf{s}}\right)$.

Ces équations sont résolues en trois étapes grâce à un schéma séquentiel explicite qui consiste à résoudre séquentiellement les étapes suivantes : interaction entre les phases, diffusion visqueuse, incompressibilité du mélange, détermination de la pression et de l'état de remplissage (Barriere et al., 2001 ; Donea et al., 1982 ; Gelin et al., 2002).

\subsection{Modélisation et simulation de l'étape de densification}

Un modèle thermo-élasto-viscoplastique décrivant le procédé de densification a été proposé (Barriere et al., 2003) pour analyser la densification de poudres par diffusion à l'état solide. La vitesse de déformation $\dot{\varepsilon}$ est composée de la vitesse de 
déformation élastique $\dot{\varepsilon}^{e}$, de la vitesse de déformation thermique $\dot{\varepsilon}^{t}$ et de la vitesse de déformation viscoplastique $\dot{\varepsilon}^{v p}$, sous la forme additive :

$$
\dot{\varepsilon}=\dot{\varepsilon}^{e}+\dot{\varepsilon}^{t}+\dot{\varepsilon}^{v p}
$$

La vitesse de déformation élastique, supposée linéaire et isotropique, s'exprime sous la forme suivante :

$$
\dot{\sigma}=D^{e} \dot{\varepsilon}^{e}=D^{e}\left(\dot{\varepsilon}-\dot{\varepsilon}^{t}-\dot{\varepsilon}^{v p}\right)
$$

où $D^{e}$ est le tenseur élastique linéaire. La vitesse de déformation thermique associée à la dilatation thermique est exprimée sous la forme suivante :

$$
\dot{\varepsilon}^{t}=\alpha \dot{T} I
$$

où $\alpha$ est le coefficient de dilatation thermique, $\dot{T}$ est l'incrément de température et $I$ est la matrice identité.

La vitesse de déformation visqueuse associée à la densification par diffusion visqueuse s'exprime grâce au modèle $G, K, \sigma_{s}$ (Song et al., 2006) sous la forme :

$$
\dot{\varepsilon}^{v p}=\frac{\operatorname{dev}(\sigma)}{2 G}+\frac{\sigma_{m}-\sigma_{s}}{3 K} I
$$

où $\operatorname{dev}(\sigma)$ est le déviateur des contraintes, $\sigma_{m}=\frac{1}{3} \operatorname{tr}(\sigma)$ est la contrainte moyenne, $G$ et $K$ sont respectivement le module de cisaillement et de compressibilité du matériau exprimés en fonction des paramètres principaux de la densification (Bordia et al., 1988). $\sigma_{s}$ est la contrainte de densification définie par $\sigma_{\mathrm{s}}=\frac{C \rho^{2}}{r}$ où $r$ est le rayon moyen des particules, $\rho$ est la densité relative du matériau poreux et $C$ est une constante à identifier. Différents modèles ont été proposés et identifiés, ils reposent généralement sur des approches expérimentales et des approches micromécaniques (Song et al., 2005 ; Blaine et al., 2003). Les différents modèles cherchent à représenter les retraits géométriques survenant lors de la densification par diffusion solide, ainsi que la porosité résiduelle après densification. Les retraits géométriques influencent la géométrie finale des 
composants, tandis que la porosité résiduelle conditionne la résistance mécanique des composants obtenus par densification, par diffusion à l'état solide.

\subsection{Résultats de simulation}

Deux solveurs éléments finis ont été développés : l'un pour la simulation de la phase d'injection en moulage par injection de poudres, l'autre pour la simulation de la phase de densification par diffusion à l'état solide, utilisant les deux modèles précédemment décrits.

a)

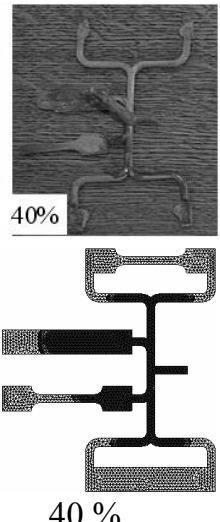

$40 \%$

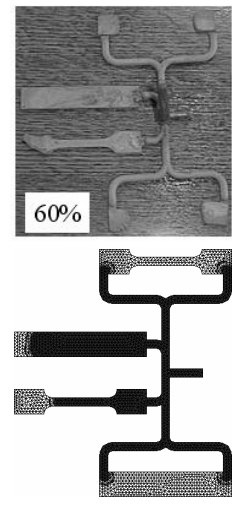

$60 \%$

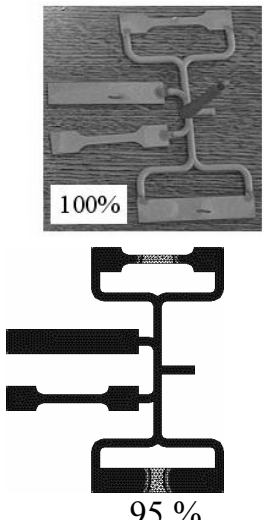

$95 \%$
Eprouvette avant densification

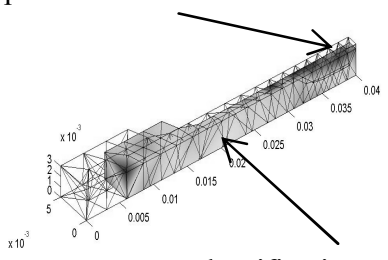

b)

Retrait obtenu numériquement: $14,8 \%$,

Densité finale obtenue numériquement : $95,7 \%$

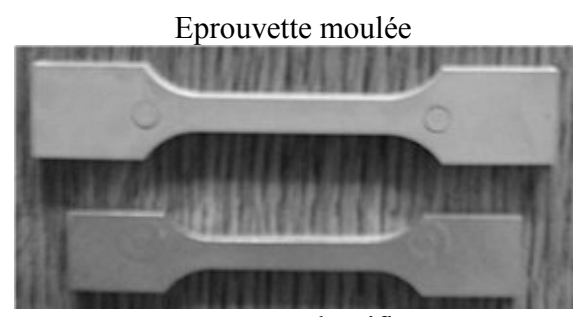

Eprouvette densifiée

Retrait obtenu expérimentalement : $14 \%$, Densité finale obtenue expérimentalement : $97 \%$

Figure 1. a) Comparaisons des fronts d'injection en cours de moulage par injection entre expérimentation et simulation. b) Comparaison des retraits et densités obtenues expérimentalement et par simulation (Barriere, 2005)

La figure 1 présente des simulations des phases d'injection et de densification réalisées sur des cas tests développés au laboratoire. Le premier cas test concerne un 
moule multicomposant comportant des éprouvettes de traction et de flexion, tandis que le second concerne un composant de type éprouvette de traction. Ces composants ont été injectés dans les empreintes de moule, puis ensuite densifiés. La figure 1a présente une comparaison entre fronts d'injection expérimentaux et fronts obtenus par simulation pour chacun des deux composants, tandis que la figure $1 \mathrm{~b}$ présente les retraits finaux obtenus après densification par diffusion à l'état solide.

\section{Optimisation du moulage par injection de poudres}

\subsection{Méthodologie d'optimisation}

L'optimisation complète du moulage par injection de poudres consiste en la détermination optimale des constituants du mélange initial (fractions volumiques de poudres et de liants, nature des liants), ainsi qu'en la détermination optimale des paramètres physiques et des cycles thermiques de l'étape de densification, figure 2, de façon à réaliser des composants répondant aux propriétés quantitatives (en termes de propriétés mécaniques et d'aspect) définies par l'utilisateur final, tout en assurant la productivité maximale. Cette chaîne d'optimisation peut dans certains cas comprendre des aspects optimisation de forme, mais également des aspects optimisation de cycles de procédés, ou encore de proportion de constituants ou de paramètres physiques et mécaniques.

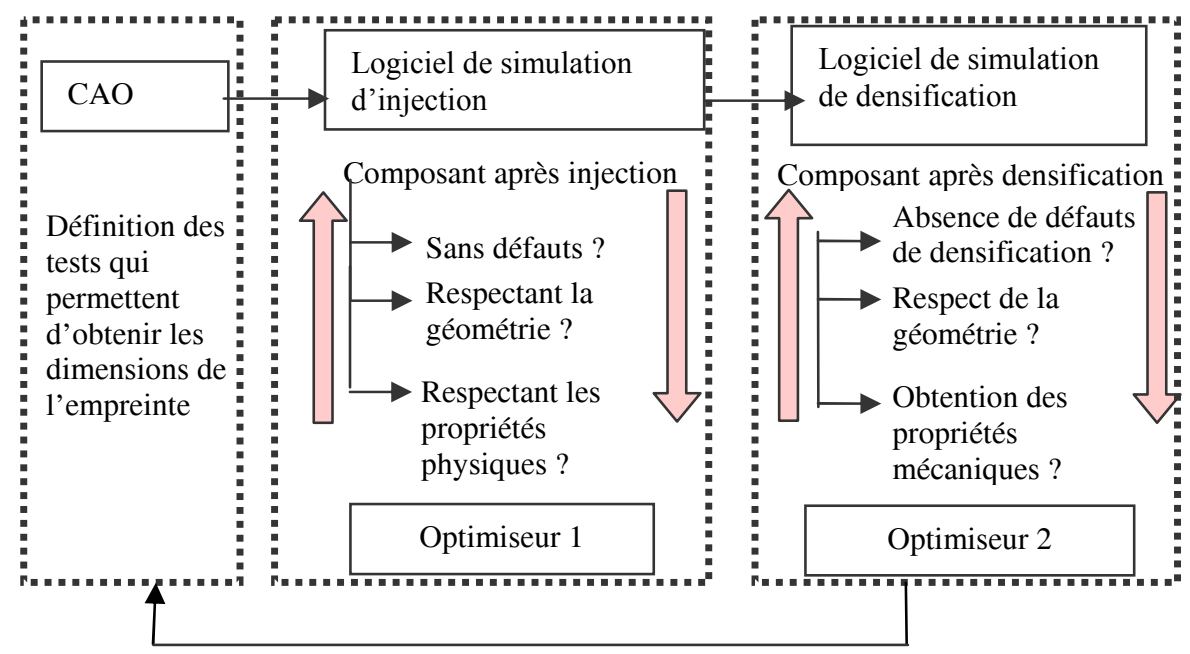

Figure 2. Diagramme de l'optimisation de la chaîne complète du procédé de moulage par injection de poudres

Pour l'optimisation de procédé, les fonctions objectifs sont soit des fonctions qualité destinées à limiter l'apparition de défauts pouvant survenir lors du procédé, 
soit des fonctions objectif en vue d'approcher les caractéristiques physiques ou mécaniques désirées. Suivant les procédés, ces différentes fonctions peuvent être regroupées dans des approches multi-objectifs permettant la combinaison pondérée de chacune des fonctions (Jozefowiez et al., 2005).

\subsubsection{Cas test utilisé}

Pour valider la procédure d'optimisation développée, on propose de traiter un exemple 3D associé à un composant correspondant à une prothèse de hanche (Liksonov, 2006), figure 3. La pose de la prothèse de hanche est une intervention chirurgicale dont le but est de remplacer une articulation défectueuse de la hanche, appelée également prothèse totale de hanche ou arthroplastie totale de hanche. La prothèse de hanche présente des caractéristiques géométriques complexes pour être produite par moulage par injection de poudres, du fait de sa longueur, de son épaisseur et de son volume. Les données expérimentales associées à ce cas test, sont issues de travaux de thèse réalisés au laboratoire (Liksonov, 2006).

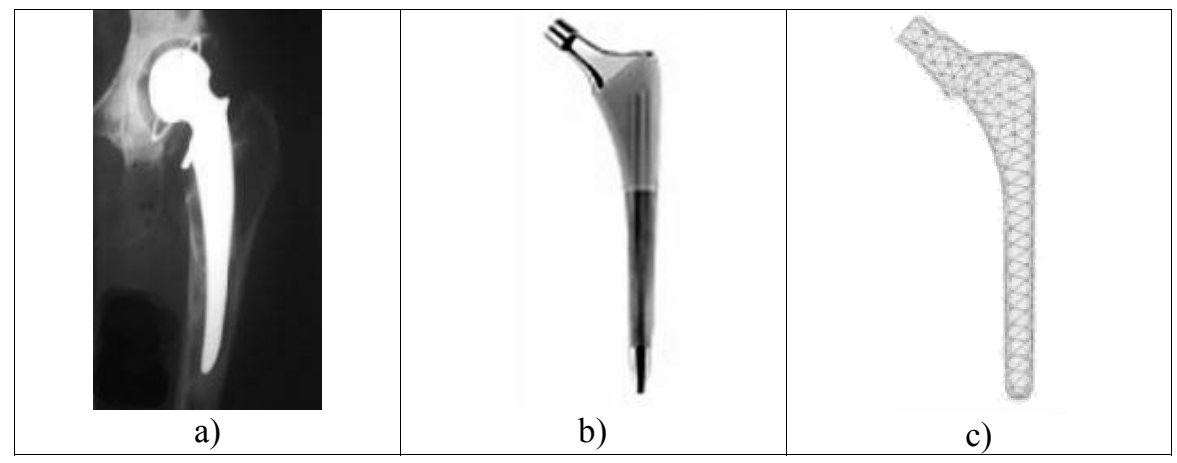

Figure 3. Cas test : a) Implant fémoral correspondant à une prothèse de hanche, b) géométrie de la prothèse, c) maillage éléments finis utilisé pour la simulation du remplissage de moule

\subsubsection{Matériau utilisé pour la prothèse de hanche}

Pour le cas test associé à la prothèse de hanche, on considère un mélange à base de poudres d'alumine $\mathrm{Al}_{2} \mathrm{O}_{3}$ qui est utilisé pour valider l'optimisation de la chaîne complète du procédé MIM. Les données matériaux concernant le mélange sont présentées dans le tableau 1. 


\begin{tabular}{|l|c|}
\hline Densité des poudres d'alumine & $3,96 \mathrm{~g} / \mathrm{cm}^{3}$ \\
\hline Densité du fluide & $1,2 \mathrm{~g} / \mathrm{cm}^{3}$ \\
\hline Fraction volumique de poudres d'alumine & 0,6 \\
\hline
\end{tabular}

Tableau 1. Données matériaux pour le mélange à base de poudres d'alumine $\mathrm{Al}_{2} \mathrm{O}_{3}$ (Quirmbach et al., 2004)

\subsection{Optimisation de la phase d'injection}

\subsubsection{Phénomènes de ségrégation}

Durant la phase d'injection, les phénomènes de ségrégation de poudres ou encore de distribution hétérogène de la fraction volumique de poudre, apparaissent (Gelin et al., 2004). Ces phénomènes correspondant à une variation importante et localisée des proportions de poudre et de liant survenant lors de l'injection. La ségrégation conduit ultérieurement dans la suite du procédé, à des distorsions géométriques importantes lors des opérations de déliantage et de densification, altérant ainsi les dimensions et les propriétés mécaniques finales des composants. La ségrégation peut intervenir lors des changements de vitesse ou de direction dans l'écoulement pendant l'injection et peut être quantifiée avec l'aide des simulations numériques de l'étape d'injection du moulage par injection de poudres métalliques. La figure 4 indique les zones plus ou moins riches en liant. Elle peut être également appréhendée grâce à des mesures expérimentales de densité par exemple (Barriere, 2005).

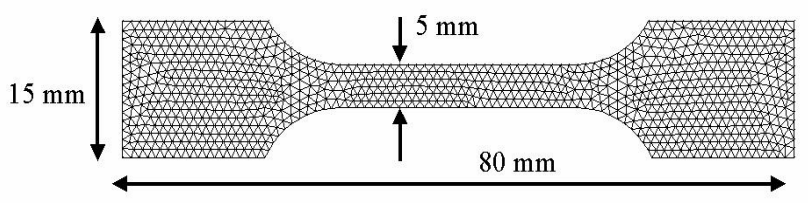

Type d'éléments: 2D MINI-éléments

Nb. d'éléments : 7100, Nb. de noeuds: 11300

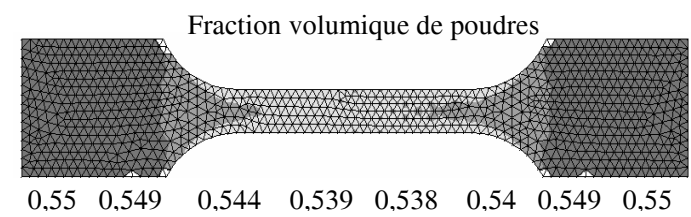

Figure 4. Maillage de l'éprouvette de traction et fraction volumique finale de poudres calculée par le modèle numérique développé par les auteurs (Gelin et al., 2004) 


\subsubsection{Fonction objectif}

Durant la phase d'injection, la fonction objectif utilisée pour l'optimisation des paramètres d'injection doit conduire à une répartition de poudre la plus uniforme qui soit dans la pièce après injection pour supprimer les effets de ségrégation mais aussi les défauts d'injection tels que les bulles d'air où la fraction volumique de poudre résultante soit alors nulle. Cette fonction objectif $F_{O B J}$ est traduite par l'équation [7].

$$
F_{O B J}(X)=\frac{1}{N} \sum_{i=1}^{N} \frac{\left|\phi_{S, i}(X)-\phi_{s_{0}}\right|}{\Phi_{s_{0}}}
$$

où $\phi_{s, i}$ est la valeur calculée de la fraction volumique de poudre au $\mathrm{i}^{\mathrm{e}}$ nœud tandis que $\phi_{s_{0}}$ est la valeur de la fraction volumique initiale de poudre, $N$ est le nombre de nœuds du maillage et $X$ est le vecteur des paramètres à optimiser (tableau 2). La norme quadratique a été utilisée pour cette approche initiale. On minimise l'écart par rapport à la valeur initiale de la fraction volumique de poudres considérée comme constante.

\begin{tabular}{|c|l|l|}
\hline Paramètres & \multicolumn{1}{|c|}{ Paramètres de procédé } & \multicolumn{1}{c|}{ Valeur } \\
\hline $\mathrm{x}_{1}$ & Loi de viscosité & Viscosité newtonienne $(5-15$ Pa.s $)$ \\
\hline $\mathrm{x}_{2}$ & Pression d'injection & {$[1-16 \mathrm{MPa}]$} \\
\hline $\mathrm{x}_{3}$ & Température du mélange & {$\left[90-220^{\circ} \mathrm{C}\right]$} \\
\hline $\mathrm{x}_{4}$ & Température du moule & {$\left[30-50^{\circ} \mathrm{C}\right]$} \\
\hline $\mathrm{x}_{5}$ & $\begin{array}{l}\text { Coefficient d'interaction entre } \\
\text { poudres et le liant }\end{array}$ & {$\left[0,001-0,007\right.$ Pa.s.m $\left.{ }^{-2}\right]$} \\
\hline $\mathrm{x}_{6}$ & Densité de poudres & {$\left[1-8 \mathrm{~g} / \mathrm{cm}^{3}\right]$} \\
\hline $\mathrm{x}_{7}$ & Densité du fluide & {$\left[0,5-2 \mathrm{~g} / \mathrm{cm}^{3}\right]$} \\
\hline $\mathrm{x}_{8}$ & $\begin{array}{l}\text { Fraction volumique initiale } \\
\text { de poudres }\end{array}$ & {$[0,4-0,7]$} \\
\hline
\end{tabular}

Tableau 2. Les paramètres de procédé à optimiser pour la phase de moulage par injection 


\subsubsection{Proposition d'une méthode d'optimisation}

Le but est d'obtenir une distribution de poudres la plus uniforme qui soit dans le composant, à l'aide d'optimiseurs bien adaptés au logiciel utilisé pour la simulation de l'étape d'injection. Cet objectif correspond à une répartition uniforme de poudre à chaque nœud de maillage du composant. Cet objectif est difficilement accessible si l'on utilise des méthodes d'optimisation de type directe, compte tenu du fait que l'évaluation de la fonction objectif nécessite de très nombreux calculs par éléments finis.

On propose donc de décomposer le problème d'optimisation en trois étapes, à savoir :

- identification des paramètres les plus significatifs (Modèle de TAGUCHI pour les plans d'expériences) (Schimmerling et al., 1998),

- approximation de la fonction objectif par la méthode des surfaces réponses utilisant l'interpolation des moindres carrés mobiles (MLS) (Nayroles et al., 1992 ; Belytschko et al., 1996),

- utilisation d'un algorithme génétique couplé aux surfaces réponses pour évaluer les paramètres optimaux (GENOCOP3) (Vergara et al., 2002).

Dans la première étape de la méthodologie développée, la méthode des plans d'expériences est utilisée de façon à repérer les paramètres qui ont une influence significative sur la valeur de la fonction objectif, sans prendre en considération la nature physique du mélange, c'est-à-dire identifier les paramètres via l'influence de la composition (poudres + liants) sur la ségrégation.

L'utilisation d'un plan factoriel complet à 2 niveaux pour une première évaluation de paramètres de ce problème d'optimisation demanderait $256\left(2^{8}\right)$ évaluations de la fonction objectif (calculs éléments finis), ce qui représente un temps de résolution assez important.

De façon à réduire ce temps, un plan factoriel fractionnaire de 16 expériences basé sur le tableau $\mathrm{L}_{16}\left(2^{15}\right)$ de Taguchi est utilisé (Pillet, 1997). Une étude de la variance liée au test de Snedecor-Fisher de la réponse (l'évaluation de la fonction objectif) par rapport aux facteurs a été par ailleurs utilisée dans le but d'identifier les facteurs significatifs, en évaluant la contribution des coefficients sur la réponse du modèle de Taguchi à partir de la normalisation de leur valeur par rapport à la somme des carrés des écarts des réponses.

Cette méthode a permis de mettre en évidence les quatre paramètres les plus influents : la fraction volumique initiale de poudre, le coefficient d'interaction, les masses volumiques de poudres et du fluide.

Une méthode d'optimisation a été proposée (Ayad et al., 2004), s'appuyant sur les plans d'expérience permettant de choisir les paramètres influents sur la ségrégation et les surfaces réponses adaptatives, pour construire une approximation satisfaisante de la fonction objectif, qui permet d'accélérer la localisation des optima (figure 5). 


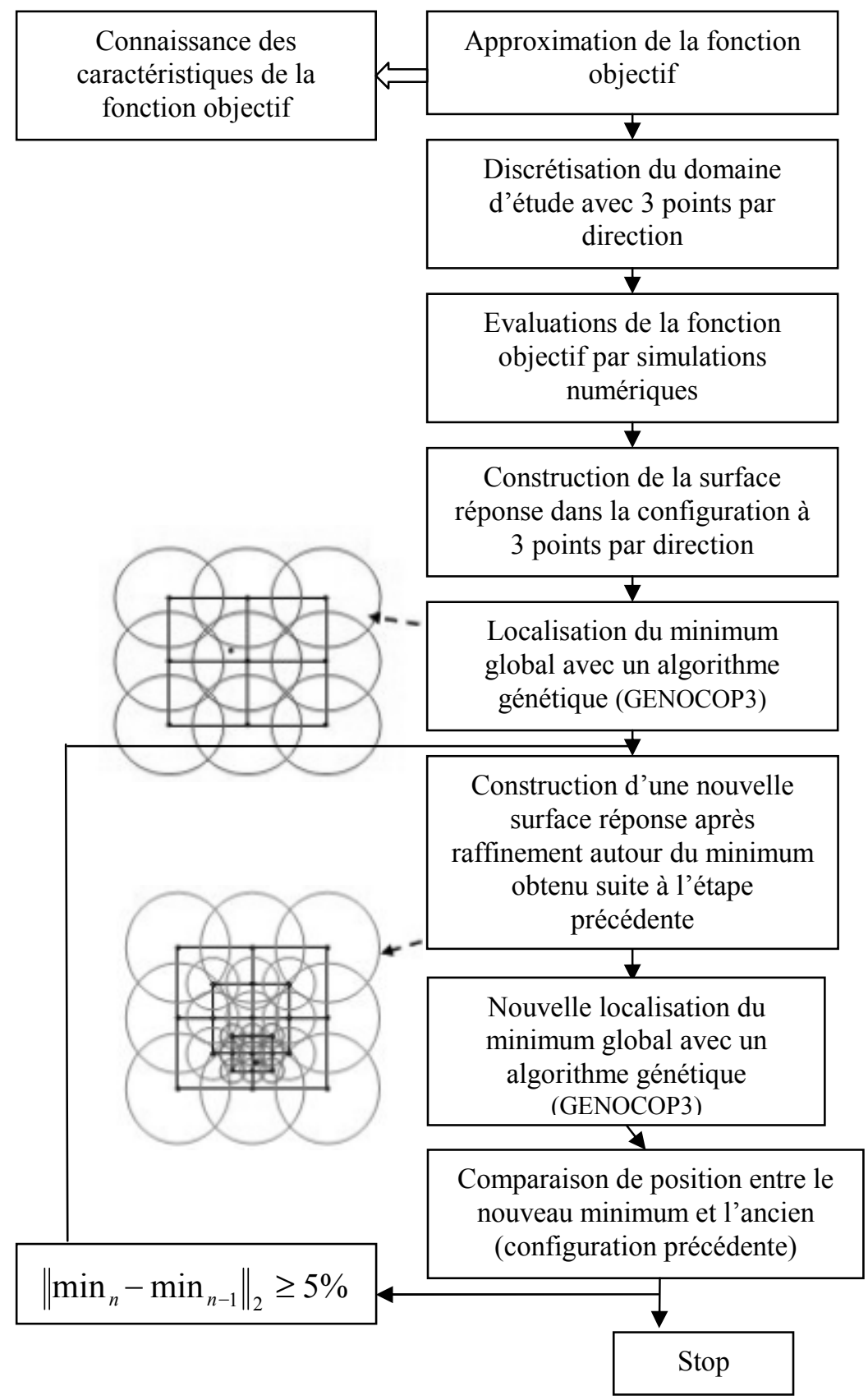

Figure 5. Organigramme de la discrétisation paramétrique de l'espace adaptatif par création de nouveaux éléments 
La démarche initiale de construction de la surface réponse avec la méthode des moindres carrés mobiles est la suivante : créer un domaine de dimension égale au nombre de paramètres, discrétiser le domaine, générer les éléments diffus et résoudre le système matriciel associé à la configuration d'éléments existante.

Dans une première étape, cette approche a été appliquée avec les 4 paramètres les plus influents sur la fonction objectif avec une discrétisation comprenant 3 points par direction c'est-à-dire 9 évaluations de la fonction objectif (ou 4 par direction c'est-à-dire 16 évaluations de la fonction objectif).

Ensuite, un algorithme génétique est utilisé pour localiser le point optimal global de l'approximation de la fonction objectif pour cette configuration initiale.

Une fois que l'on a localisé le point optimal sur la surface réponse, on cherche l'élément dont le centre est le plus proche de ce point et qui en même temps le contient. Au droit de cet élément, on créé des nouveaux éléments, dont les centres sont à une distance égale à la moitié de la distance entre deux nœuds de la configuration initiale. Le rayon de chaque nouvel élément, ainsi que de celui qui contient le point d'optimisation, sera égal à la moitié du rayon des éléments initiaux.

Après avoir obtenu une nouvelle surface réponse dans l'espace paramétrique, on résout le nouveau système matriciel avec tous les éléments existants, dont la solution permet d'obtenir une approximation plus précise de la fonction objectif par rapport à la configuration précédente, en particulier dans la région qui contient les nouveaux éléments ajoutés. Ce processus se répète jusqu'au moment où le point optimal obtenu suite à deux itérations consécutives reste dans une tolérance préalablement défini $(5 \%)$, figure 5 .

\subsubsection{Application de la méthodologie sur l'éprouvette de traction obtenue par moulage par injection}

La méthodologie proposée basée sur la méthode des surfaces réponses est comparée avec l'optimisation directe, en utilisant un algorithme génétique.

Dans cette application, la méthode des surfaces réponses adaptatives est appliquée à l'éprouvette de traction. L'optimum est donné tableau 3, celui-ci est obtenu après 480 évaluations de la fonction objectif.

\begin{tabular}{|c|c|c|}
\hline $\begin{array}{c}\text { Méthode } \\
\text { d'optimisation }\end{array}$ & $\begin{array}{c}\text { Nombre } \\
\text { d'évaluations }\end{array}$ & Valeurs optimales des paramètres à optimiser \\
\hline MLS adaptative & 480 & $\begin{array}{l}\phi_{S_{0}}=0,505, \rho_{f}=2,12 \mathrm{~g} / \mathrm{cm}^{3}, \\
\rho_{p}=3,55 \mathrm{~g} / \mathrm{cm}^{3}, \mathrm{k}=0,0015 \text { Pa.s. } \mathrm{m}^{-2} .\end{array}$ \\
\hline
\end{tabular}

Tableau 3. Résultats obtenus par l'approche adaptative 
Les valeurs obtenues à partir de la méthode des surfaces réponses adaptatives couplée à la méthode des moindres carrés mobiles sont proches de celles obtenues par l'algorithme génétique, avec une différence de l'ordre de $8 \%$. Les résultats d'optimisation obtenus par cette approche sont comparés avec les résultats des autres méthodes: la méthode des surfaces réponses standard et la méthode basée sur l'utilisation directe d'un algorithme génétique, voir tableau 4.

\begin{tabular}{|l|c|c|c|}
\hline $\begin{array}{l}\text { Méthode } \\
\text { d'optimisation }\end{array}$ & $\begin{array}{c}\text { Erreur comparée } \\
\text { par rapport à } \\
\text { l'algorithme } \\
\text { génétique }\end{array}$ & $\begin{array}{c}\text { Nombre } \\
\text { d'évaluations }\end{array}$ & Temps de calcul \\
\hline $\begin{array}{l}\text { Algorithme } \\
\text { génétique }\end{array}$ & Référence & 3000 & 2,5 jours \\
\hline $\begin{array}{l}\text { MLS (4 points par } \\
\text { direction) }\end{array}$ & $21 \%$ & 256 & 3 heures \\
\hline MLS adaptative & $8,12 \%$ & 480 & 6,5 heures \\
\hline
\end{tabular}

Tableau 4. Comparaison des résultats obtenus grâce à l'algorithme génétique, les méthodes MLS et MLS adaptatives

La comparaison de la distribution de la fraction volumique de poudres dans l'éprouvette après injection, obtenue grâce à l'optimisation par les trois méthodes (algorithme génétique, MLS et MLS adaptative) est représentée en figure 6. On remarque clairement l'intérêt et l'amélioration sur la détermination des paramètres optimaux résultant de la méthode adaptative proposée.

Après validation de la méthodologie proposée et comparaison entre les résultats des trois optimiseurs appliqués à une éprouvette de traction, le cas test industriel 3D correspondant à une prothèse de hanche est traité, en utilisant la méthode des surfaces réponses adaptatives. 
Algorithme génétique

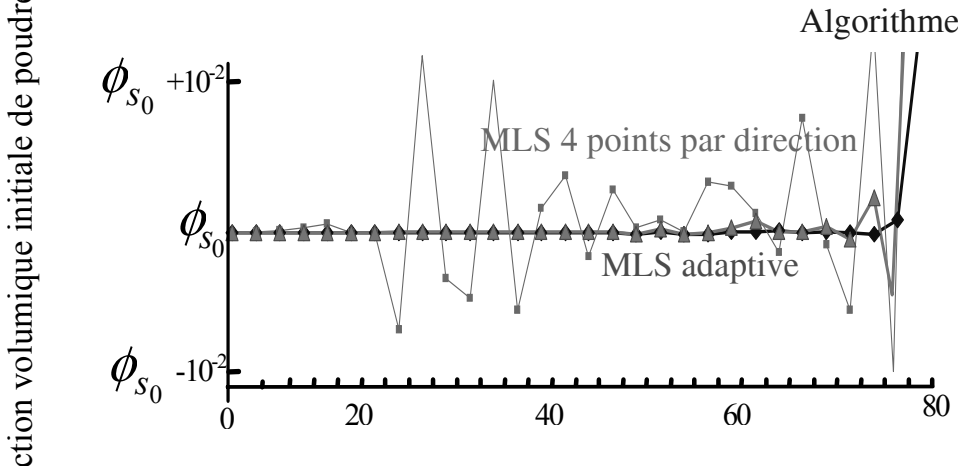

Axe de l'éprouvette de traction $(\mathrm{mm})$

Figure 6. Comparaison entre les distributions de poudres le long de l'axe de l'éprouvette de traction, obtenues par utilisation des trois méthodes d'optimisation

3.2.5. Application à l'optimisation des paramètres d'injection pour la prothèse de hanche

Pour le cas test associé à la prothèse de hanche, deux paramètres ont été optimisés : la pression d'injection [10-20 MPa] et la fraction volumique initiale de poudres [0,4-0,7]. Le mélange utilisé pour la prothèse considérée est à base de poudres d'alumine. Les résultats associés à cette optimisation sont présentés tableau 5 et figure 7 . Le remplissage de la cavité de moule associée à la prothèse se fait d'une manière uniforme et progressive comme indiqué figure 8, et la répartition de poudres devient nettement plus uniforme après optimisation.

\begin{tabular}{|l|c|c|}
\hline $\begin{array}{c}\text { Méthode } \\
\text { d'optimisation }\end{array}$ & $\begin{array}{c}\text { Nombre } \\
\text { d'évaluations }\end{array}$ & $\begin{array}{c}\text { Paramètres résultant de } \\
\text { l'optimisation }\end{array}$ \\
\hline $\begin{array}{l}\text { MLS + méthode } \\
\text { adaptive }\end{array}$ & 22 & $\phi_{S_{0}}=0,4, P_{i n j}=20 \mathrm{MPa}$ \\
\hline
\end{tabular}

Tableau 5. Résultats de la procédure d'optimisation utilisée pour l'injection de la prothèse de hanche 
412 REMN - 17/2008. Optimisation des procédés de mise en forme

La figure 7a indique les points où la fonction objectif (figure 7b) a été évaluée en utilisant la méthode MLS adaptative.

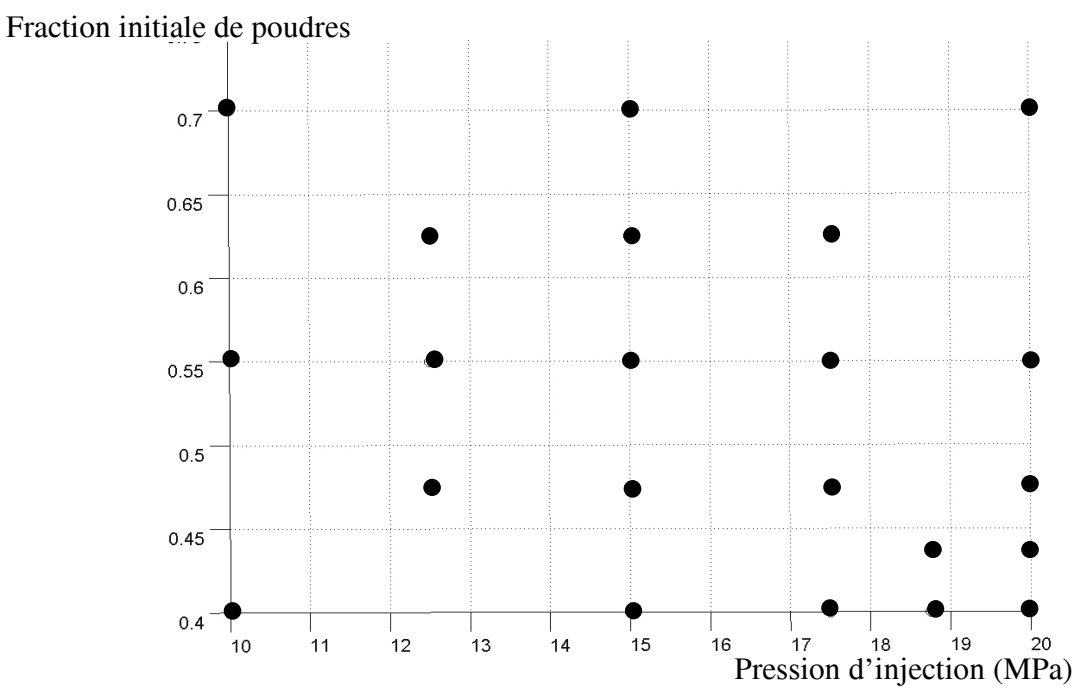

a)

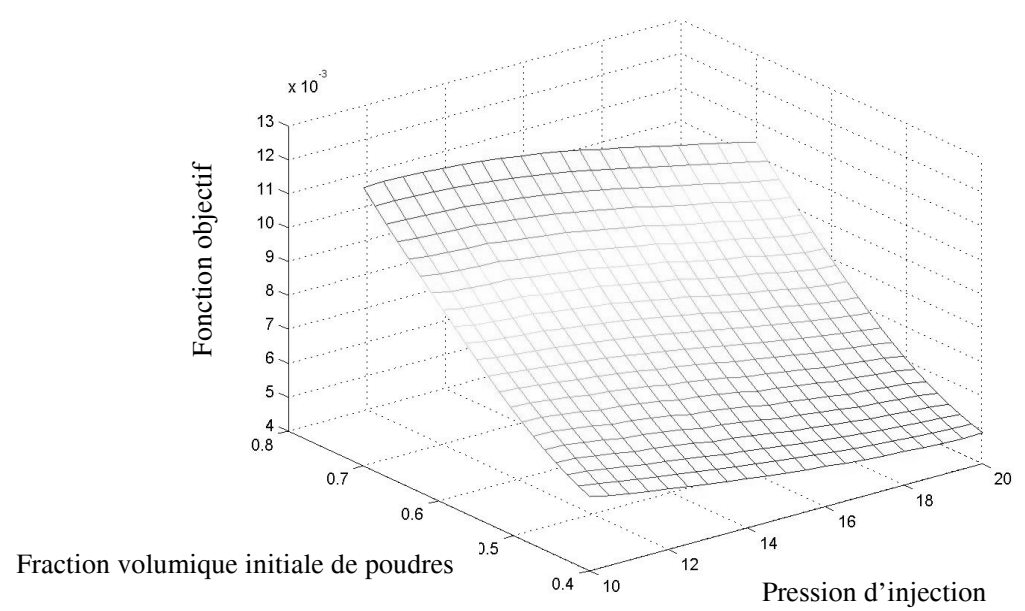

b)

Figure 7. a) Position des points dans l'espace paramétrique pour l'optimisation de la fonction objectif, b) surface réponse obtenue par la méthode MLS adaptative en fin d'étape d'optimisation 
Après le contrôle de la ségrégation, une méthode basée sur l'identification paramétrique des modèles macroscopique est utilisée pour la prévision du retrait et la détermination de la taille de l'empreinte du moule.

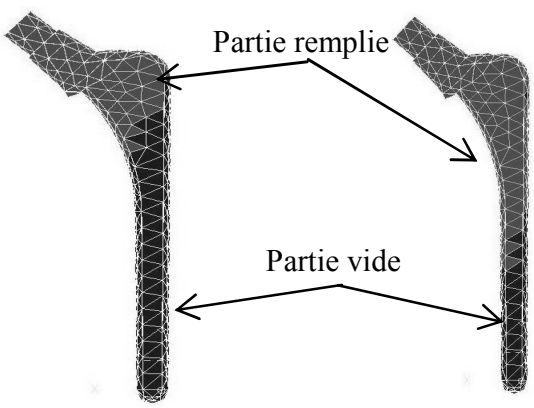

$50 \%$
$75 \%$

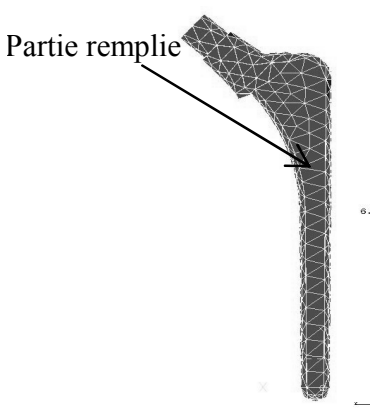

$95 \%$

Figure 8. Fronts de remplissage après optimisation

Au niveau de l'éprouvette de traction et pour une géométrie de moule donnée, les paramètres les plus significatifs pour minimiser la ségrégation sont les paramètres définissant la composition du mélange initial : la fraction volumique de poudre initiale, la densité des poudres et du liant et le coefficient d'interaction entre la poudre et le liant. Dans le cas de l'injection de la prothèse de hanche, le paramètre procédé le plus important correspond à la pression d'injection.

\section{Optimisation de l'étape de densification}

\subsection{Modèles macroscopiques}

Les approches macroscopiques utilisées pour la détermination des paramètres $G$, $K$ et $\sigma_{s}$ du modèle de densification, reposent sur des études à l'échelle microscopique, avec des hypothèses permettant les calculs analytiques. Le modèle de (McMeeking et al., 1992) prend en compte la diffusion aux joints de grains et la diffusion volumique comme source de transport de la matière. La diffusion volumique est modélisée en faisant des hypothèses importantes sur les chemins qu'empruntent les lacunes. La diffusion se fait entre particules sphériques de taille homogène. Le modèle suppose en outre que le nombre de contacts entre les sphères varie de manière linéaire, en fonction de $d$. 
La théorie conduit aux expressions suivantes de $G, K$, et $\sigma_{s}$ :

$$
\begin{aligned}
& G=\left(\frac{d_{0}}{d}\right)^{\frac{2}{3}}\left(\frac{d-d_{0}}{1-d_{0}}\right)^{2} \frac{d r_{0}{ }^{3}}{90 D_{i f f}(T)} \\
& K=\left(\frac{d_{0}}{d}\right)^{\frac{2}{3}}\left(\frac{d-d_{0}}{1-d_{0}}\right)^{2} \frac{d r_{0}{ }^{3}}{54 D_{i f f}(T)} \text { et } D_{i f f}(T) \frac{\Omega \delta D_{b 0} e^{-\frac{Q_{b}}{R T}}}{k T} \\
& \sigma_{s}=\frac{8 \gamma_{s}}{r_{0}}\left(\frac{d_{0}}{d}\right)^{\frac{1}{3}} d
\end{aligned}
$$

dans les expressions $[8,9,10] \quad r_{0}$ représente le rayon initial des particules sphériques, $\gamma_{s}$ est la densité d'énergie surfacique sur une frontière solide-vapeur, $d_{0}$ est la densité initiale (rapport entre le volume de matière et le volume total), $\delta D_{b 0}$ est un coefficient de diffusion, $d$ est la densité volumique de la matière dense, $\Omega$ est le volume atomique, $T$ est la température, $R$ est la constante des gaz parfaits, $Q_{b}$ est l'énergie d'activation, $k$ est la constante de Boltzmann. Les paramètres que l'on cherche à identifier dans la présente étude sont $Q_{b}, \delta D_{b_{0}}, \gamma_{s}, r_{0}$ et $\Omega$.

\subsection{Approche et méthode d'identification}

Une méthode d'identification inverse a été développée pour évaluer les paramètres de densification pour un mélange à base de poudres d'alumine $\mathrm{Al}_{2} \mathrm{O}_{3}$. La réponse numérique pour un essai de dilatation est comparée à la courbe expérimentale, une procédure d'optimisation est utilisée afin de minimiser l'écart entre les deux courbes, permettant d'identifier le jeu de paramètres choisis. La procédure d'optimisation est basée sur la minimisation de la fonction objectif suivante :

$$
\left\{\begin{array}{l}
\min _{x}\left[F(x)=\sqrt{\sum_{i=1}^{M}\left(\frac{f_{i, e}(x)-f_{i, s}(x)}{f_{e}(x)}\right)^{2}}\right] \\
x=\left[\delta D_{b_{0}}, Q_{b}, \gamma_{s}, r_{0}, \Omega\right]
\end{array}\right.
$$

où $M$ est le nombre de relevés expérimentaux pour différentes valeurs du temps de cycle, i est le ie relevé, $f_{i, e}(x)$ est le retrait mesuré expérimentalement par l'essai de dilatométrie, $f_{i, s}(x)$ est le retrait résultant de la simulation, $F(x)$ représente la différence au sens des moindres carrés entre la courbe expérimentale et la courbe numérique et $x$ représente le vecteur des paramètres à identifier. 
La méthode d'identification des paramètres de la loi viscoplastique visqueuse est appliquée à la densification par diffusion à l'état solide pour un mélange à base de poudres d'alumine $\mathrm{Al}_{2} \mathrm{O}_{3}$. La courbe de dilatation utilisée est issue de la littérature (Opferman et al., 1998) (figure 9).

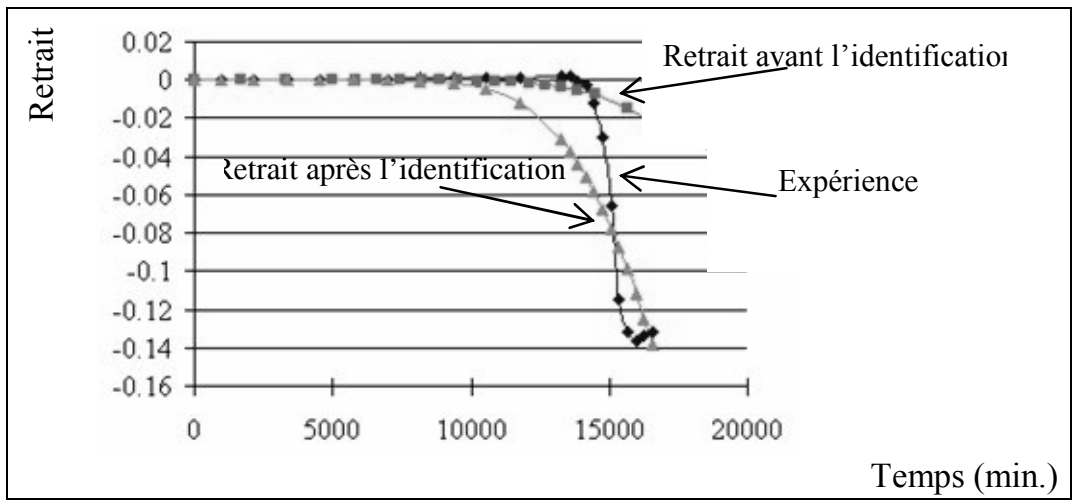

Figure 9. Comparaison entre valeurs expérimentales (Opferman et al., 1998) et valeurs identifiées par la procédure proposée

Le jeu de données initiales utilisées pour un mélange à base de poudres d'alumine $\mathrm{Al}_{2} \mathrm{O}_{3}$ ainsi que les résultats d'identification paramétrique sont donnés tableau 6. Les paramètres matériaux $Q_{b}, \delta D_{b_{0}}, \gamma_{s}, r_{0}$ et $\Omega$ sont pris en compte dans la procédure d'identification. La courbe obtenue avec ce jeu de paramètres initiaux est déterminée avec la procédure décrite en section pécédente.

\begin{tabular}{|c|c|c|}
\hline Paramètres & Valeurs avant identification & Valeurs finales \\
\hline$Q_{\mathrm{b}}(\mathrm{KJ} / \mathrm{mol})$ & 384,0 & 327,5 \\
\hline$\delta D_{\mathrm{b} 0}\left(\mathrm{~m}^{3} / \mathrm{s}\right)$ & $8,6 \times 10^{-10}$ & $7,30 \times 10^{-10}$ \\
\hline$\gamma_{s}\left(\mathrm{~J} / \mathrm{m}^{2}\right)$ & 0,70 & 0.80 \\
\hline$r_{0}(\mathrm{~m})$ & $7,0 \times 10^{-6}$ & $5,1 \times 10^{-6}$ \\
\hline$\Omega\left(\mathrm{m}^{3}\right)$ & $4,2 \times 10^{-29}$ & $5,0 \times 10^{-29}$ \\
\hline
\end{tabular}

Tableau 6. Comparaison entre valeurs initiales et identifiées pour le modèle de densification 
Au niveau de l'identification des paramètres matériaux $Q_{b}, \delta D_{b_{0}}, \gamma_{s}, r_{0}$ et $\Omega$ cités dans les équations [9] et [10] du modèle de McMeeking-Kuhn, le paramètre le plus sensible et important $Q_{b}$ du fait qu'il intervient dans une fonction exponentielle.

\subsection{Validation de la simulation de l'étape de densification appliquée à une prothèse de hanche}

Les validations du modèle de densification et de la procédure d'identification sont réalisées sur le cas test correspondant à la prothèse de hanche dont le matériau constitutif est un mélange de poudre d'alumine $\mathrm{Al}_{2} \mathrm{O}_{3}$. La simulation de la densification est conduite avec les paramètres de la loi de comportement identifiée pour ce mélange (tableau 6). Le cycle de température utilisé pour les simulations éléments finis consiste en une montée en température à une vitesse de $5^{\circ} \mathrm{C} / \mathrm{min}$ jusqu'à $1400^{\circ} \mathrm{C}$.

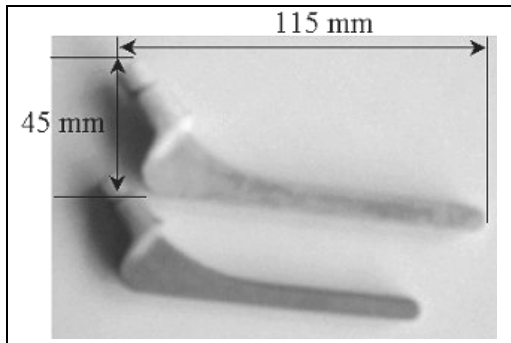

a) Prothèse moulée et densifiée obtenue expérimentalement (Liksonov, 2006)

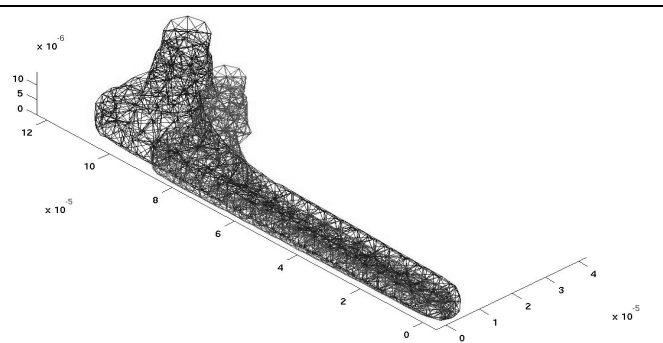

b) Comparaisons de forme entre la prothèse après moulage et après densification, obtenues par simulation

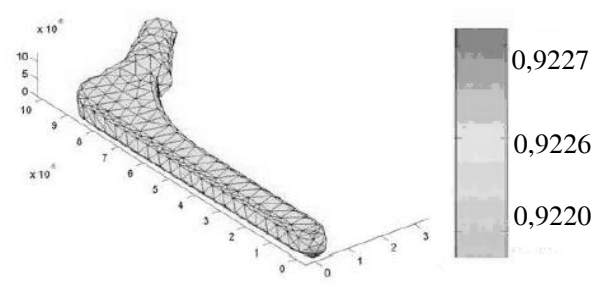

c) Densité finale de la prothèse de hanche

Figure 10. a) Comparaison de la forme entre prothèse moulée et densifiée obtenue expérimentalement, b) prothèses moulées et densifiées obtenues par simulation c) densité finale de la prothèse densifiée 
Des mesures géométriques ont été réalisées sur cette prothèse et un retrait d'environ $13 \%$ a été mesuré (voir figure 10a). Une densité expérimentale finale de $97 \%$ a été obtenue.

Les géométries de la prothèse obtenues par simulation éléments finis après déliantage et densification sont illustrées figure 10b. Les contours de densités finales sur la prothèse densifiée sont illustrés figure 10c. Un retrait moyen de 15,2 \% est obtenu, la densité finale atteint une valeur de $92,2 \%$ résultant de la simulation. Celle-ci est moins importante que celle obtenue par expérimentalement. Une amélioration des résultats numériques peut être obtenue grâce à la prise en compte des conditions de posage sur les supports de densification et l'identification grâce à une fonction multiobjectif des paramètres de densification générant le retrait.

\section{Optimisation complète du procédé}

\subsection{Méthodologie}

Pour l'optimisation de la chaîne complète, une première simulation de la phase d'injection est réalisée en utilisant les dimensions finales de la pièce afin d'obtenir une première évaluation de la fraction volumique de poudres après injection, pour notamment pouvoir simuler la phase de densification et obtenir ainsi une prédiction du retrait, qui sera ensuite utilisée pour évaluer les dimensions de l'empreinte à l'aide d'une transformation de type homothétie appliquée sur le volume. Une nouvelle simulation de la chaîne complète est ensuite effectuée avec ces nouvelles dimensions. Cette procédure itérative est répétée jusqu'à l'obtention des dimensions souhaitées dans une tolérance fixée (figure 11).

La méthodologie proposée est itérative à deux étapes :

1) la première étape de la première itération consiste à réaliser la simulation complète du procédé : simulation de la phase d'injection avec les paramètres d'injection optimisés et simulation de la phase de densification par diffusion solide avec la distribution de fraction volumique de poudres obtenue en fin d'injection à partir des dimensions du composant final à obtenir,

2) la deuxième étape consiste à ajouter les dimensions de l'empreinte du moule pour corriger les effets du retrait de densification. Pour ce faire un cœfficient d'homothétie est calculé à partir du retrait final en fin de densification associé à l'étape 1. Le retrait $r$ est évalué au moyen de l'expression:

$$
r=\underset{i}{\operatorname{Moy}}\left(\frac{d_{i}-d_{f}}{d_{i}}\right)
$$


où $d_{i}$ est la distance initiale entre tout nœud $i$ du maillage par rapport à un nœud encastré choisi comme nœud de référence, tandis que $d_{f}$ est la distance après densification.

Le centre d'homothétie est le nœud encastré et le cœfficient d'homothétie est défini par $\frac{1}{1-r}$.

Les dimensions de l'empreinte du moule sont définies par la transformation d'homothétie et une nouvelle simulation complète des phases d'injection et de densification est réalisée.

Ensuite les dimensions du composant densifié sont comparées aux dimensions souhaitées, et la procédure est répétée itérativement jusqu'à l'obtention des dimensions souhaitées, dans une tolérance définie initialement.

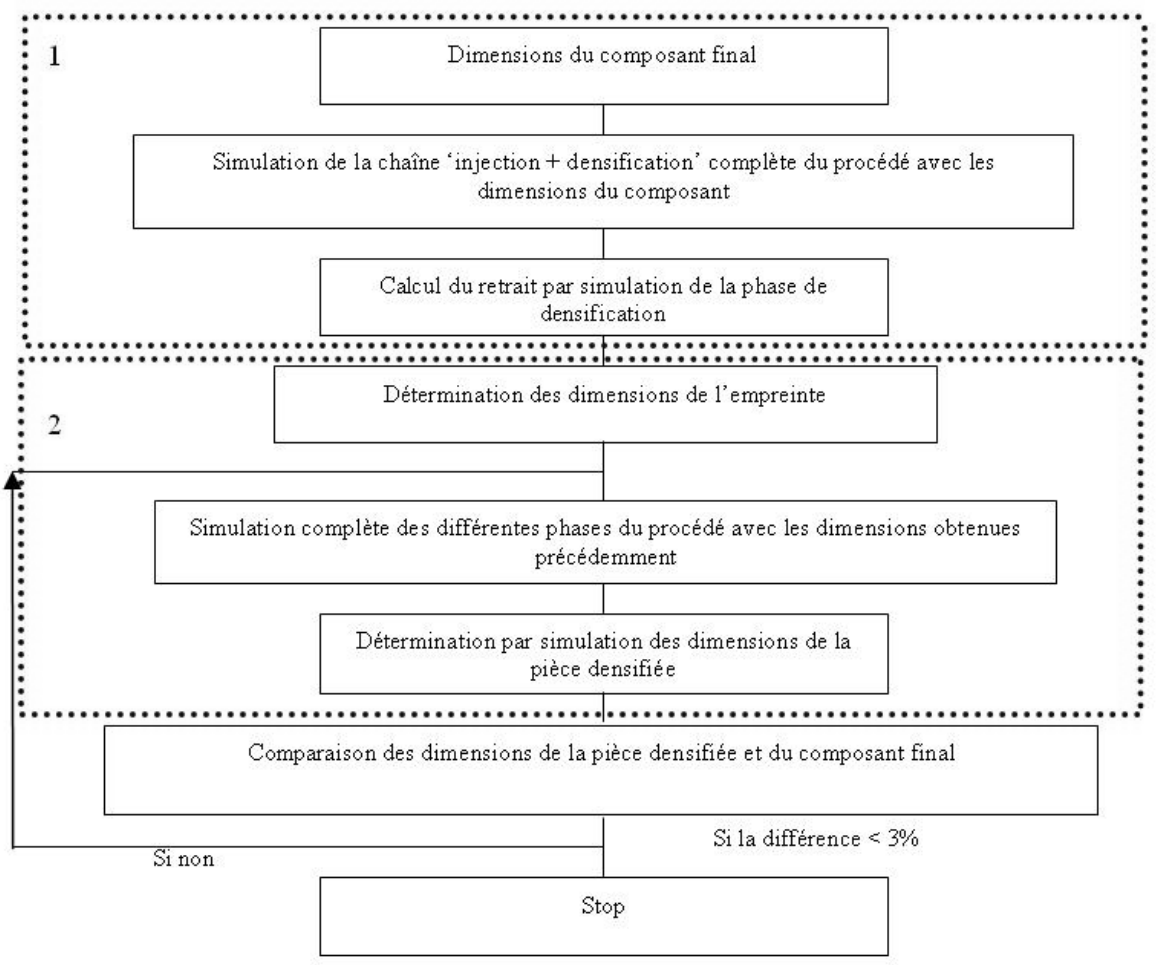

Figure 11. Algorithme utilisé pour la prédiction des dimensions de l'empreinte du moule 


\subsection{Résultats}

La méthodologie d'optimisation de forme proposée dans la section précédente est appliquée au cas de la prothèse de hanche. A la fin du procédé MIM, on souhaite obtenir une prothèse densifiée dont les dimensions géométriques sont indiquées figure 12 .

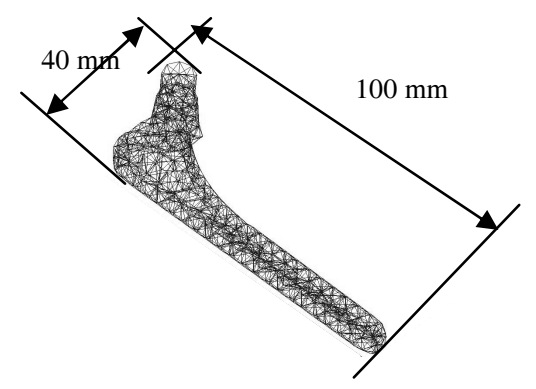

Figure 12. Caractéristiques géométriques souhaitées de la prothèse

Les résultats obtenus pour la prothèse représentée figure 12 en suivant la méthodologie proposée sont indiqués figure 13. Après trois itérations, le retrait se stabilise à 15,2\% permettant ainsi de déterminer les dimensions des empreintes du moules.

L'optimisation complète du procédé de moulage par injection de poudres, comprenant à la fois les étapes du moulage par injection, puis de densification par diffusion, a été réalisée afin de déterminer les dimensions de la cavité du moule, permettant de garantir la géométrie du composant souhaité, ainsi que de minimiser la possible ségrégation de poudres. Les résultats obtenus sur la prothèse de hanche confirment la faisabilité et la pertinence de l'approche proposée.

\begin{tabular}{|l|l|}
\hline 10 & $\begin{array}{l}1^{\text {re }} \text { itération : } \\
\text { - simulation de l'étape d'injection } \\
\text { avec les dimensions finales de la } \\
\text { pièce, } \\
\text { - simulation de l'étape de } \\
\text { densification, } \\
\text { - retrait de } 15,5 \% .\end{array}$ \\
\hline
\end{tabular}




\begin{tabular}{|l|l|l|}
\hline & & $\begin{array}{l}2^{\mathrm{e}} \text { itération : } \\
- \text { homothétie de coefficient de } \\
1,155, \\
- \text { simulations de l'étape d'injection } \\
\text { et de densification, } \\
\text { - retrait de } 15,21 \% .\end{array}$ \\
\hline
\end{tabular}

Figure 13. Illustration synoptique de la procédure complète de simulationoptimisation développée pour la détermination automatique des dimensions d'empreintes de moule

\section{Conclusions}

Plusieurs approches d'optimisation et d'identification sont décrites dans ce papier. La première approche d'optimisation, basée sur la méthode des surfaces réponses adaptatives concerne l'optimisation de la phase d'injection d'un mélange de poudres et de liants polymères dans une empreinte de moule. Les résultats obtenus indiquent la possibilité d'optimiser les paramètres d'injection et de réduire les phénomènes de ségrégation. La seconde approche proposée concerne l'identification paramétrique d'un modèle de densification par diffusion solide utilisé 
pour la simulation de l'étape de densification du moulage par injection de poudres. Les résultats obtenus sont également très satisfaisants et permettent par combinaison séquentielle des optimisations de la phase d'injection et de la phase de densification, l'optimisation globale du procédé.

La démarche retenue, consistant, à partir d'une géométrie de moule correspondant aux dimensions finales du composant à obtenir, de corriger par approximations successives les dimensions de celui-ci, pour aboutir à une géométrie garantissant le respect des dimensions finales du composant permettent ainsi de garantir le respect des dimensions, mais aussi celui des propriétés mécaniques après densification par diffusion solide.

\section{Bibliographie}

Ayad G., Lejeune A., Barriere T., Gelin J.C., "Finite element modeling and optimization of powder segregation during metal injection molding", J. of Steel Grips, 2, 2004, p. 393-398.

Ayad G., Contribution à l'optimisation multi-physiques et multi-étapes du moulage par Injection de Poudres, Thèse de l'Université de Franche-Comté, 2006, p. 1-156.

Barriere T., Gelin J.C., Liu B., “Analysis of phase segregation effects arising in fluid-particle flows during metal injection molding", Int. J. of Forming Process, Hermes Science, vol. 4, n 3-4, 2001, p. 199-216.

Barriere T., Gelin J-C., Dvorak P., Renault D., «Expérimentation, modélisation et simulation numérique par éléments finis en injection et densification de poudres céramiques ", $\sigma^{e}$ Colloque National en Calcul des Structures, Giens, 2003, p. 141-148.

Barriere T., Physique et technologie du Moulage par Injection de Poudres, Mémoire d'Habilitation à Diriger des Recherches, Université de Franche-Comté, 2005, p. 1-131.

Belytschko T., Krongauz K., Organ D., Fleming M., Krysl P., "Meshless methods: an overview and recent developments", Comput. Methods Appl. Mech Engrg, vol. 139, 1996, p. 3-47.

Bordia R.K., Scherer G.W., "On constrained sintering-I Constitutive model for a sintering body", Acta Metall., vol. 36, n 9, 1988, p. 2393-2397.

Donea J., Giuliani S., Laval H., Quartapelle L., "Finite element solution of the unsteady Navier-Stokes equations by a fractional step method", Comp. Meth. in Appl. Mech. and Engrg., vol. 30, 1982, p. 53-73.

Dvorak P., Barriere T., Gelin J.C., "Jetting in Metal Injection Molding of 316 L Stainless Steel”, Powder Metallurgy, Maney Publishing, vol. 48, 2005, n³ 3, p. 254-260.

Gelin J.C., Barriere T., Liu B., "Mould design methods by experiment and numerical simulation in metal injection molding", J. of Engineering Manufacture, John Wiley \& Sons, Part B, vol. 126, 2002, p. 1533-1547.

Gelin J.C., Barriere T., «Ségrégation de phases dans les écoulements de polymères fondus chargés en poudres métalliques », Mécanique et Industries, vol. 5, 2004, p. 429-440. 
German R.M., Sintering Theory and Practice, Wiley-Interscience, 1996.

German R.M., Bose A., Injection Moulding of Metals and ceramics, Princeton, New Jersey, USA, 1997.

Jozefowiez N., Frédéric Semet F., Talbia E-G., "The bi-objective covering tour problem", J. of computers and operations research, 2005.

Liksonov D., Experimental and numerical study of the manufacturing and behaviour of femoral THR components produced by injection molding of fiber composite and by powder technologies, Thèse de l'Université de Franche-Comté, 2006, p. 1-140.

McMeeking R.M., Kuhn T., "A diffusional creep law for powder compacts", Acta Metallurgica Materiala, vol. 40, 1992, p. 961-969.

Nayroles B., Touzot G., Villon P., La Méthode des Eléments Diffus, Compte rendu à l'Academie de Sciences, 313, série II, Paris, France, 1991, p. 133-138.

Opfermann J., Blumm J., Emmerich W.D., "Simulation of sintering behavior of a ceramic green body using advanced thermokinetic analysis", Thermochimica Acta, vol. 318, 1998, p. 213-220.

Piccirillo N., Lee D., "Jetting in powder Injection Molding”, Advanced in powder Metallurgy, vol. 2, 1991, p. 119-126.

Pillet M., Les Plans d'Expériences par la Méthode TAGUCHI, Les Editions d'organization, 1997.

Quirmach P., Schartz S., Magerl AD., "The application of injection moulding technology in modern tablewareproduction", CFI Ceramirc Forum International, vol. 3, 2004, p. 1-5.

Schimmerling P., Sisson J.C., Zaïdi A., Pratique des plans d'expérience, Lavoisier, 1998.

Song J., Ayad G., Barriere T., Gelin J.C., Liu B., Renault D., "Modelling, Simulation and simulation and identification of solid state sintering after metal injection moulding", Proceeding of $8^{\text {th }}$ ESAFORM conference on Material Forming, vol. 1, 2005, p. 171-174.

Song J., Gelin J.C., Barriere T., Liu B., "Experiments and numerical modelling of solid state sintering for $316 \mathrm{~L}$ stainless steel components", Journal of Materials Processing Technology, vol. 177, 2006, p. 352-355.

Vergara F.E., Khouja M., Michalewicz Z., “An evolutionary algorithm for optimizing material flow in supply chains", Computers \& Industrial Engineering, vol. 3, 2002, p. 407-421. 